\title{
New Surgical Technique for The Optic Disc Pit Associated with Macular Hole
}

\section{Settimio Rossi, Francesco Maria D’Alterio, Beatrice Gallo, Francesco Testa, Francesca Simonelli and Michele Della Corte}

Eye Clinic, Multidisciplinary Department of Medical, Surgical and Dental Sciences, University of Campania Luigi Vanvitelli, Via Sergio Pansini 5, Naples 80131, Italy

*Corresponding author: Michele Della Corte, Eye Clinic, Multidisciplinary Department of Medical, Surgical and Dental Sciences, University of Campania Luigi Vanvitelli, Via Sergio Pansini 5, Naples 80131, Italy, Tel: +39 081566 6444; E-mail: michele.dellacorte@unicampania.it

Rec Date: May 10, 2017, Acc Date: June 26, 2017, Pub Date: June 28, 2017

Citation: Rossi S, D’Alterio FM, Gallo B, Testa F, Simonelli F, et al. (2017) New Surgical Technique for The Optic Disc Pit Associated with Macular Hole. Med Case Rep 3:3.

\section{Abstract}

Background: Optic disc pit is a congenital abnormality of the optic nerve head amaculopathy can develop in $25 \%$ to $75 \%$ of cases and usually confers a poor visual prognosis as a result of a longstanding serous macular detachment, formation of macular hole, and atrophy of the retinal pigmented epithelium. At the moment, there is not a single treatment that is universally accepted.

Method: A 65-year-old Caucasian man underwent a complete ophthalmological examination.

Results and discussion: Ophthalmoscopic examination and macular Optical Coherence Tomography showed in the right eye an optic disc pit maculopathy complicated by full thickness macular hole. Multifocal-Electroretinogram showed microvolted responses in all the regions. We performed in the right eye a 25 gauge pars plana triamcinolone-assisted vitrectomy with an inverted internal limiting membrane flap inserted both in the macular hole and in the optic disc pit. At 3 months from surgery the patient showed an improvement of macular structure and of visual function as well.

Conclusion: We report a case in which a "new" surgical approach was used in a patient affected by optic disc pit maculopathy complicated with a full thickness macular hole. Our technique was effective in improving the maculopathy and leading to an appreciable increase of the visual function; in particular, we found not only a significant improvement of visual acuity, but also of electrophisyological responses, considered by previous studies very sensitive in assessing functional alterations in this disease.

Keywords: Maculopathy; mfERG; Optic disc pit; Full thickness macular hole; Inverted internal limiting membrane flap

\section{Introduction}

Optic disc pit (ODP) is a congenital anomaly of the optic nerve head, unilateral in most of cases, although it can be bilateral in $15 \%$ of cases; it was first described in 1882 by Wiethe as a gray, whiteyellow or black round depression of the optic disc [1]. The estimated incidence of ODP is of about 1:11,000 people [2].

ODP is most frequently observed in the temporal portion of the optic disc [3]. Given the limits of the existing instruments in allowing an in vivo evaluation of a such excavated optic disc, most of the previous ODP studies were histopathological. They showed that ODP is a herniation of dysplastic retina into a rich in collagen pocket extending posteriorly through a defect of the lamina cribrosa, often into the subarachnoid space [4]. Hence, a communication between intraocular and extraocular spaces is created.

ODP can be either an isolated anomaly or in association with an optic disc coloboma. In fact, these two anomalies may have a common pathologenetic mechanism: both diseases probably occur as a result of an incomplete closure of the optic fissure during the embryonic development of the primitive epithelial papilla $[3,5]$.

Halbertsma was the first who described the association of ODP with a macular lesion and poor visual acuity, which is actually named ODP maculopathy (ODP-M) [6]. ODP-M can develop in $25-75 \%$ of patients in the third or fourth decade of life; it causes a poor visual prognosis, with a final visual acuity of $6 / 60$ or worse, resulting from the longstanding serous macular detachment and the possible development of a macular hole with subsequent atrophy of the retinal pigmented epithelium $[2,5,7]$. Optical coherence tomography (OCT) allowed us to detect ODP-M and to study its morphology. It consists of retinoschisis-like separations of the retinal layers followed by the development of a serous macular detachment [8-11].

ODP-M treatment is controversial as there is not an universally accepted strategy of management. ODP-M resolves spontaneously in $25 \%$ of cases, so the initial recommendation is a conservative management or the use of an oral corticosteroid $[12,13]$. But the poor visual outcome of the 
spontaneously-resolved macular detachment has encouraged the development of surgical approaches $[12,13]$. Many authors have suggested different treatment strategies including: intravitreal gas injection with or without laser photocoaugulation of the temporal edge of the optic disc; vitrectomy with gas tamponade; vitrectomy and induced posterior vitreous detachment with or without internal limiting membrane (ILM) peeling and laser photocoaugulation [13-15]. All these strategies aim at the reabsorption of the intra and subretinal liquid and at the creation of a scar in the retina surrounding the pit.

We report a case of a new surgical approach used for a patient affected by ODP-M complicated with a full thickness macular hole (FTMH).

\section{Case Report}

This study was carried out in compliance with the Declaration of Helsinki.

A 65-year-old Caucasian man was seen at the Eye Clinic of the University of Campania Luigi Vanvitelli, Naples, Italy. The patient referred a progressive reduction of visual acuity in both eyes (the worst eye was the right one) over the last few years. In particular, he had undergone cataract surgery in his right eye that was not followed by a visual recovery. He did not have an ocular history of eye trauma or inflammatory diseases; he was affected by sistemic hypertension and type II diabetes mellitus.

At examination, the patient had a BVCA of $20 / 600$ in the RE and 20/80 in the LE. Biomicroscopy of the anterior segment revealed a transparent cornea, pseudophakia in RE and cataract in LE, normally pigmented iris and normal intraocular pressure in both eyes. The ophthalmoscopic examination showed a white-yellow oval depression in the optic disc localized in the temporal segment associated with macular dystrophy in RE and a normal fundus in LE.

Macular Optical Coherence Tomography (OCT) showed in the $\mathrm{RE}$ a neuroretinal detachment in the nasal side of the macula, with a FTMH and a coexisting retinal schisis around the lesion and an epiretinal membrane (Figure 1 ) and in the LE a normal foveal thickness and depression.

Multifocal-Electroretinogram (mfERG) was performed in order to test the retinal function and the probability of functional recovery. It showed microvolted responses in all the regions in the RE (Figure 1 ) and normal responses in all regions in the LE.

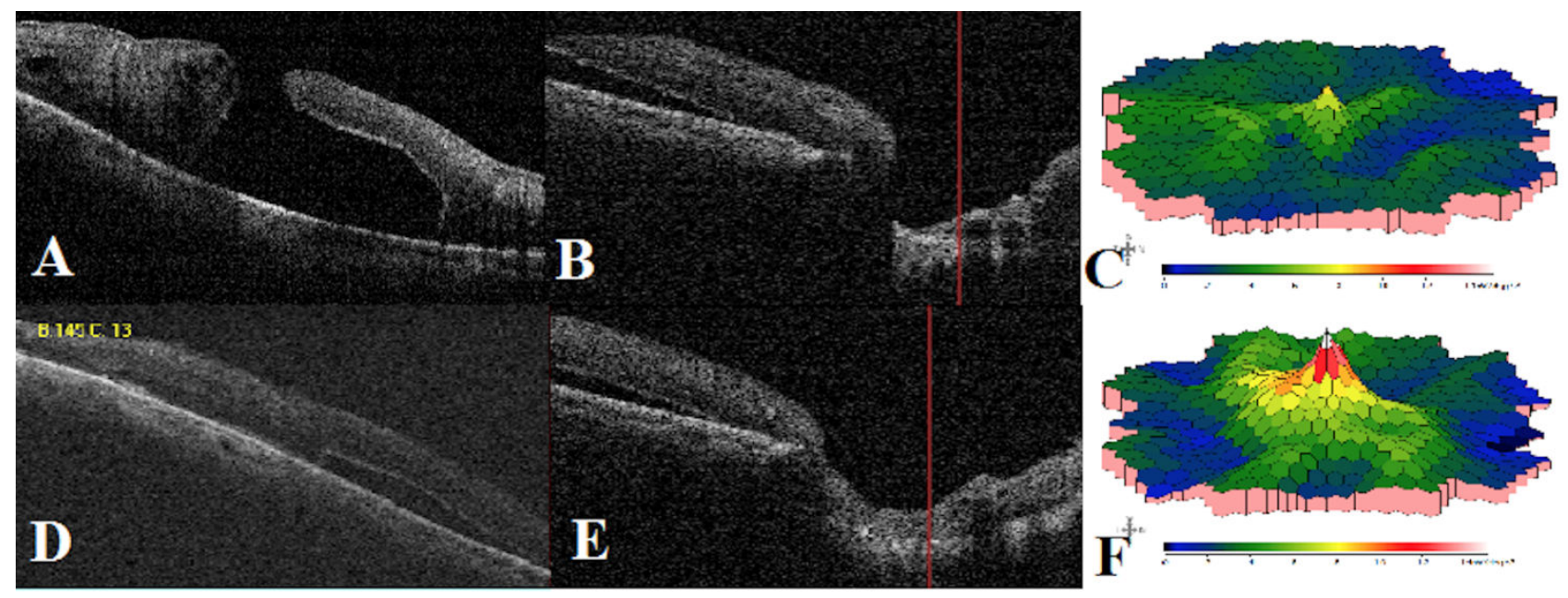

Figure 1 At the baseline: (A) Macular OCT showed neuroretinal detachment in nasal side of the macula with FTMH and with a coexisting retinal schisis round the lesion and epiretinal membrane in RE; (B) Optic Disc OCT showed ODP; (C) mfERG showed microvolted signals in all regions in RE. After 3 months, (D and E) macular OCT showed the closure of FTMH and reduction of neuroretinal detachment, optic disc OCT confirm the presence of ILM flap in the FTMH and in the ODP; (F) mfERG exam showed normal signal in central rings and sub-normal signal in peripheral rings.

Thanks to these tests we made a diagnosis of Optic Disc Pit (ODP) complicated in his RE by maculopathy (ODP-M) and full thickness macular hole (FTMH).

We performed in the RE a $25 \mathrm{G}$ pars plana triamcinoloneassisted vitrectomy, with induction of posterior hyaloid detachment and removal of epiretinal membranes; then, we performed a balanced salt solution (BSS)/air exchange and a ILM staining with retidyne (Lutein and bryllant blue) and used perfluorocarbonate to stabilize the posterior pole; then, we removed the ILM with the maculorexis technique and made an inverted flap in the macular hole; after that, we performed the removal of ILM at the temporal edge of the optic disc and made an inverted flap in the ODP; finally, we removed perfluorocarbonate and subsequently buffered with SF6 at $20 \%$.

The patient maintained a facedown position for the entire day after surgery and then 4 hours daily for the subsequent 6 days. 
We followed-up the patient at 1 and 3 months from surgery. In the LE the BCVA, biomicroscopy, ophthalmoscopic examination, OCT and mf-ERG did not change during the follow-up.

In the RE at 1 month from surgery the patient had a BCVA of 20/70; the fundus examination and the OCT showed a closure of the FTMH and a reduction in the height of the neuroretinal detachment. The mfERG showed a normal signal in the central rings and a subnormal signal in the peripheral rings.

At 3 months from surgery the BCVA in the RE improved at 20/50; the fundus examination and the OCT confirmed the closure of the FTMH and a further reduction of the neuroretinal detachment. MfERG showed a normal signal in the central rings and a subnormal signal in the peripheral rings.

\section{Discussion}

Congenital ODP is a rare disease $(1: 11.000)$, unilateral in most of cases and often associated with maculopathy (25\% to $75 \%$ of cases) being the main cause of visual loss [2].

The pathogenesis of macular detachment occurring in ODP is still unclear; four mechanisms to explain the fluid's origin were proposed: from the vitreous cavity, from leaky blood vessels at the base of the pit, from the subarachnoid space and from the orbital space surrounding the dura [1]. Many authors speculated that the retinal elevation communicating with optic disc pit consists initially of a separation between the inner retinal layers later followed by a splitting of the outer layers. Some authors also have hypothesized that vitreous tractional forces play a role in the pathogenesis of ODP-M [11,12]. FTHM, rarely associated with ODP-M, may be the evolution of ODP-M caused by similar vitreous tractional forces, resulting in degeneration of neuroretina and photoreceptor loss $[4,7]$.

The poor visual prognosis of untreated ODP-M has encouraged various surgical techniques, among which: laser photocoagulation of the temporal margin of the optic disc, pars plana vitrectomy with or without use of an internal tamponade, and internal drainage of submacular fluid [12-14]. Vitrectomy with removal of posterior hyaloid, alone or combined with C3F8 gas injection, and laser photocoaugulation to the temporal edge of optic disc are successful in reattaching the macula [5], even though laser has some side effects like paracentral scotomas and others visual field defects [4]. The use of fibrin glue to close the optic nerve pit is another proposed treatment strategy [16].

However, up to now an optimal treatment for ODP-M does not exist, as no one is better than the others and because of the unclear pathogenesis and the rarity of the disease.

In our case, we performed a vitrectomy with posterior hyaloid removal and inverted internal limiting membrane (ILM) flap placed both in the FTMH and in the ODP, 20\% SF6 gas tamponade followed by facedown positioning. We did not perform laser to the temporal edge of the disc. The technique of ILM inverted flap is a technique recently described by Michalewska et al. [17], whose rationale is that a segment of peeled-off ILM left attached to the retina may induce gliosis both in the retina and on the surface of the ILM and may be also a scaffold for tissue proliferation. We also performed a peeling sparing the fovea (maculorexis) to reduce the surgical stress of this region [18]. Another target was to create a barrier between the pit and the retinal space, thus preventing the fluid from passing through the pit. For this reason, to close the congenital defect of the lamina cribrosa we placed the ILM flap in the pit [19], being a more natural tissue compared to fibrin. We employed $20 \%$ SF6 endotamponade and facedown positioning to reduce the risk of post-operative flap dislocation and to allow the reabsorption of subretinal fluid.

\section{Conclusion}

In conclusion, this is the first case reported in literature in which this surgical technique, a double ILM inverted flap, was used for this disease. Just at 3 months from surgery we observed a significant improvement of BCVA, a complete closure of the FTMH, a decrease of the neuroretinal detachment and an improvement in retinal function measured by mfERG [20].

The main limitation of our case report is the lack of a longer follow-up, hence further studies with long term follow-up and more patients are needed to evaluate the effectiveness of this surgical technique.

\section{References}

Wiethe T (1882) Ein Fall von angeborener Deformität der Sehnervenpapille. Arch Augenheilkd 11: 14-19.

2. Kranenburg E (1960) Crater-like holes in the optic disc and central serous retinopathy. Arch Ophthalmol 64: 912-924.

3. Reed D (1999) Congenital pits of the optic nerve. Clin Eye Vis Care 11: 75-80.

4. Ferry AP (1963) Macular detachment associated with congenital pit of the optic nerve head. Arch Ophthalmol 70: 106-117.

5. Brown GS, Shieds JA, Goldberg RE (1980) Congenital pits of the optic nerve head II. Clinical studies in humans. Ophthalmology 87:51-65.

6. Halbertsma KTA (1927) Crater-like hole and coloboma of disc associated with changes at macula. Br J Ophthalmol 11: 11.

7. Bonnet M (1991) Serous macular detachment associated with optic nerve pits. Arch Clin Exp Ophthalmol 229: 526-532.

8. Krivoy D, Gentile R, Liebmann JM, Stegman Z, Rosen R, et al. (1996) Imaging congenital optic disc pits and associated maculopathy using optical coherence tomography. Arch Ophthalmol 114: 165-170.

9. Chiu YT, Chen HY, Tsai YY, Lin JM, Chiang CC (2006) Stratus optical coherence tomography for evaluating optic disc pits associated with maculopathy before and after vitrectomy: two case reports. Kaohsiung J Med Sci 22: 229-234.

10. Brodsky MC (1994) Congenital optic disc anomalies. Surv Ophthalmol 39: 89-112.

11. Gowdar JP, Rajesh B, Giridhar A, Gopalakrishnan M, Hussain R, et al. (2015) An insight into the pathogenesis of optic disc pit- 
associated maculopathy with enhanced depth imaging. JAMA Ophthalmol 133: 466-469.

12. Sugar HS (1967) Congenital pits of the optic disc. Am J Ophthalmol 63: 298-307.

13. Yuen CH, Kaye SB (2002) Spontaneous resolution of serous maculopathy associated with optic disc pit in a child: A case report. JAAPOS 6: 330-331.

14. Snead MP, James N, Jacobs PM (1991) Vitrectomy, argon laser, and gas tamponade for serous retinal detachment associated with an optic disc pit: $\mathrm{A}$ case report. $\mathrm{Br} J$ Ophthalmol 75:381-382.

15. Georgalas I, Kouri A, Ladas I, Gotzaridis E (2010) Optic disc pit maculopathy treated with vitrectomy, internal limiting membrane peeling, and air in a 5 -year-old boy. Can J Ophthalmol 45: 189-191.

16. Al-Sabti K, Kumar N, Chow DR, Kapusta MA (2008) Management of optic disk pit-associated macular detachment with tisseel fibrin sealant. Retin Cases Brief Rep 2: 274-247.
17. Michalewska Z, Michalewski J, Adelman RA, Nawrocki J (2010) Inverted internal limiting membrane flap technique for large macular holes. Ophthalmology 117: 2018-2025.

18. Lee CL, Wu WC, Chen KJ, Chiu LY, Wu KY, et al. (2016) Modified internal limiting membrane peeling technique (maculorrhexis) for myopic foveoschisis surgery. Acta Ophthalmol 95: e128e131.

19. Mohammed OA, Pai A (2013) Inverted autologous internal limiting membrane for management of optic disc pit with macular detachment. Middle East Afr J Ophthalmol 20: 357-359.

20. Rossi S, De Rosa G, D'Alterio FM, Orrico A, Banfi S, et al. (2016) Intrafamilial heterogeneity of congenital optic disc maculopathy. Ophthalmic Genet 8: 1-6. 